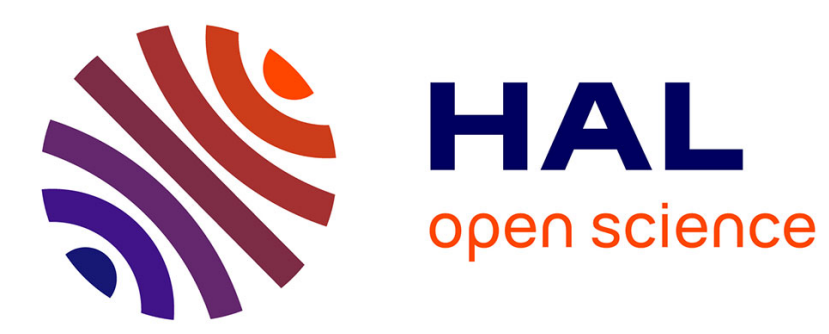

\title{
Micromechanics and macromechanics of carbon nanotube-enhanced elastomers
}

\author{
Sabine Cantournet, M.C. Boyce, A.H. Tsou
}

\section{To cite this version:}

Sabine Cantournet, M.C. Boyce, A.H. Tsou. Micromechanics and macromechanics of carbon nanotubeenhanced elastomers. Journal of the Mechanics and Physics of Solids, 2007, 55 (6), pp.1321-1339. 10.1016/j.jmps.2006.07.010 . hal-00149329

\section{HAL Id: hal-00149329 \\ https://hal.science/hal-00149329}

Submitted on 25 May 2007

HAL is a multi-disciplinary open access archive for the deposit and dissemination of scientific research documents, whether they are published or not. The documents may come from teaching and research institutions in France or abroad, or from public or private research centers.
L'archive ouverte pluridisciplinaire HAL, est destinée au dépôt et à la diffusion de documents scientifiques de niveau recherche, publiés ou non, émanant des établissements d'enseignement et de recherche français ou étrangers, des laboratoires publics ou privés. 


\title{
Micromechanics and Macromechanics of Carbon Nanotube Enhanced Elastomers
}

\author{
S. Cantournet ${ }^{a, b, *}$ M. C. Boyce ${ }^{\text {b }}$, A. H. Tsou ${ }^{c}$. \\ ${ }^{a}$ Ecoles des Mines de Paris/CNRS, Centre des matériaux/UMR 7633, BP 87, 91003 Evry, \\ France \\ ${ }^{\mathrm{b}}$ Departement of Mechanical Engineering, Massachusetts Institute of Technology, \\ Cambridge, MA, 02139 USA \\ ${ }^{c}$ ExxonMobil Chemical Company, 52000 Baytown drive, Baytown, TX 77520, USA
}

\begin{abstract}
The effects of carbon nanotubes on the mechanical behavior of elastomeric materials is investigated. The large deformation uniaxial tension and uniaxial compression stress-strain behaviors of a representative elastomer are first presented. This elastomer is then reinforced with multi-wall carbon nanotubes (MWNTs) and the influence of weight fraction of MWNTs on the large deformation behavior of the resulting composite is quantified. The initial stiffness and subsequent strain-induced stiffening at large strains are both found to increase with MWNT content. The MWNTs are also found to increase both the tensile strength and the tensile stretch at break. A systematic approach for reducing the experimental data to isolate the MWNT contribution to the strain energy of the composite is presented. A constitutive model for the large strain deformation behavior of MWNT-elastomer composites is then developed. The effects of carbon nanotubes are modeled via a constitutive element which tracks the stretching and rotation of a distribution of wavy carbon nanotubes. The MWNT strain energy contribution is due to the bending/unbending of the initial waviness and provides the increase in initial stiffness as well as the retention and further enhancement of the increase in stiffness with large strains. The model is shown to track the stretching and rotation of the CNTs with macroscopic strain as well as predict the dependence of the macroscopic stress-strain behavior on the MWNT content for both uniaxial tension and uniaxial compression.
\end{abstract}

Key words: Elastomers, nanotubes, anisotropic, nonlinear behavior PACS:

\footnotetext{
* Corresponding author. Tel: +33-160-76-30-52; Fax: +33-160-76-31-50

Email address: sabine. cantournet @ensmp. fr (S. Cantournet).
} 


\section{Introduction}

Elastomeric materials have long been reinforced by stiff fillers such as carbon black and silica particles in order to provide increased stiffness and strength, hysteresis, as well as electrical conductivity (e.g., Guth (1945), Harwood and Payne (1966), Dannenberg (1975), Kilian (1994), Bergstrom and Boyce (1999, 2000)). Typical volume fractions lie in the range of 0.10 to 0.40 . These property enhancements are invariably accompanied by a decrease in extensibility and tensile strain to failure.

The introduction of carbon nanotubes to the library of potential fillers offers new possibilities to further tailor the properties of elastomers via blending with relatively low volume fractions of fillers. Here, we explore the influence of multi-wall carbon nanotubes (MWNTs) on the mechanical behavior of a representative elastomer.

Over the past decade, there has been an explosion in research investigating the stiffness and strength enhancements that carbon nanotubes potentially offer as fillers in polymers (e.g. Baughman et al. (2002), Coleman et al. (2006)). For example, the elastic modulus of single and multi-wall carbon nanotubes is approximately $1 \mathrm{TPa}$ (e.g., Wong et al. (1997)) and, hence, relatively small volume fractions could potentially provide tremendous increases in stiffness over that of the polymer matrix (see, Shaffer and Windle (1999), Qian et al. (2000), Safadi et al. (2002), Dalmas et al. (2005),). Such improvements depend on good dispersion and alignment of the nanotubes as well as excellent bonding between the nanotube and the matrix (e.g., Chen et al. (2000) and Cooper et al. (2002)). Although there has been some success in dispersing and aligning nanotubes, these issues have not been universally resolved and bonding is still a limiting issue. The research has also primarily focused on the stiffness and strength enhancement of thermoplastics with limited attention to the influence of carbon nanotubes on elastomeric behavior. Aspects of alignment and bonding which may be beneficial to optimally enhancing thermoplastic properties may actually be detrimental to enhancing elastomeric mechanical behavior.

Elastomeric applications generally require and make use of the large deformation extensibility and resilience of the elastomer. Upon incorporation of stiff fillers into elastomers, it is generally desired to enhance the stiffness (i.e., enhance the initial stiffness and retain this stiffness enhancement for overall large strain deformation behavior) while also retaining the important attributes of large strain resilient behavior and large strain-to-break. The alignment (in terms of both overall alignment as well as waviness of the nanotubes) and bonding detriments encountered when incorporating MWNTs into thermoplastics may be used to advantage in their incorporation into elastomers. The waviness that leads to minimal stiffness enhancement in glassy and/or semicrystalline polymers (see, for example, Bradshaw et al. (2003)) can still provide sufficient enhancement to the stiffness and strength of 
the comparatively very low modulus elastomer. The property enhancement can be achieved through the unbending (straightening out) of the MWNTs with deformation such that the extensibility of the elastomer can be retained. Furthermore, if stiffness enhancement primarily results from unbending of the waviness of CNTs as opposed to axial straining of the CNTs (which relies on good bonding and shear lag load transfer from the matrix to the CNT), the stiffness enhancement will not be lost with large strains. Experiments on elastomeric-like matrices enhanced with CNTs have been reported, for example, by Frogley et al. (2002), Dufresne et al. (2002), Koerner et al. (2005) , and very recently Chen et al. (2006).The Frogley study reports on silicone-based elastomers filled with single wall CNTs (SWNTs) and carbon nanofibers (CNFs). The Frogley study reveals a dramatic increase in initial stiffness with small fractions of SWNTs, however, this stiffness enhancement is lost after only 10-20 percent strain whereupon the tangent stiffness of the nanocomposites returns to that of the parent elastomer due to debonding of the CNTs from the matrix; the tensile strain-to-break was found to decrease significantly with increase in volume fraction of either CNTs or CNFs. The Dufresne study report on a cast latex-MWNT composite and found increased stiffness with increasing MWNT content, retained stiffness to large strains, but reduced failure strains with increase in MWNT content. The Koerner and the Chen studies report on thermoplastic elastomer polyurethanes (TPUs) enhanced with CNTs where the Koerner study produced thin sheets using a casting process and the Chen study produced extruded, melt-drawn fibers thereby using processing to help achieve axial alignment of the CNTs. It is important to note that TPUs exhibit elastomeric-like behavior where, due to their copolymer structure, the TPU mechanical behavior characteristically exhibits a relatively stiff small strain region followed by a rollover at a relatively low stress level (typical of order of a few MPa to tens of MPa) to a more compliant elastomeric-like stress-strain behavior. Both the Koerner study and the Chen study report an increase in the very initial modulus with increasing CNT weight fraction, as well as an increase in the rollover flow stress; the post-rollover tangent stiffness is found to parallel that of the neat polymer, losing the dramatic stiffness enhancement seen at small strains. The Koerner study reports maintaining the same magnitude of tensile strain-to-break as the parent TPU (with a modest decrease in strain-to-break with increasing weight fraction) and the Chen study reports modest increase in strain-to-break for the lower weight fraction cases and a decrease in strain-to-break for the highest weight fraction of $17.7 \mathrm{wt}$ percent. These studies demonstrate aspects of the enhancements that can be achieved when CNTs are incorporated into elastomeric materials. However, the loss in stiffness seen at larger strains suggests that CNT/matrix configurations which do not rely on straight well-bonded CNTS may be more beneficial for elastomeric applications than for thermoplastic applications. This is demonstrated in some of the very recent work of Ahir and Terentjev (2005) and Ahir et al. (2006) where MWNTs are incorporated in a silicone-based elastomer to produce an IR-induced actuating polymer. In the Ahir studies, the CNTs are in a wavy configuration. While only limited mechanical data is presented, the stiffness is found to increase with increasing volume fraction of CNT and this stiffness enhancement is maintained with increasing strain. This 
aspect of the mechanical behavior thus contributes to the resilient and reversible behavior needed for actuation mechanisms. There is great potential for enhancing the mechanical performance of elastomers via incorporation of small weight fractions of CNTs; the multifunctional possibilities of further using such enhancements to produce actuators is enormous (note the Koerner study was directed at creating CNT-TPU actuating materials). Hence the need for quantifying and predicting the large strain multiaxial stress-strain behavior of CNT-enhanced elastomers with microstructurally-informed constitutive models will provide a tool for further designing and optimizing materials for a wide range of applications (mechanical and otherwise).

In this paper, we study the mechanics of MWNT-elastomer composites. A series of material compositions with a range in weight fraction of MWNTs are produced and evaluated in large deformation uniaxial tension and compression testing. The contribution of the MWNTs to the deformation is then evaluated in a systematic reduction of the strain energy contributions of the elastomer matrix and the MWNTs to the overall composite strain energy. A three-dimensional constitutive model is then developed and shown to capture the dependence of the large deformation stress -strain behavior of the elastomer nanocomposites subjected to different types of deformation (uniaxial tension and uniaxial compression).

\section{Experimental Program}

\subsection{Materials}

The elastomer is a brominated polymer derived from a copolymer of isobutylene and paramethylstyrene (PMS) with a trademark name of EXXPRO (or BIMSM in accordance to ASTM) (Powers et al. (1992)). Specifically, EXXPRO 3745 from ExxonMobil Chemical Company with 1.2 mole \% bromine was the BIMSM elastomer used. This BIMSM elastomer has a specific density of 0.92 and is known to possess a good combination of properties including temperature resistance, heat stability, low permeability, high damping and ozone/weathering resistance (Kresge and Wang (1993)), Multi-wall carbon nanotubes (MWNTs) of type PR19-PS MWNT were obtained from Applied Science. The MWNTs had a diameter range of approximately $70 \mathrm{~nm}$ to $150 \mathrm{~nm}$ as estimated from SEM and TEM micrographs.

Blends of BIMSM and MWNTs were prepared using a Banbury internal mixer directly operating at 100 RPM under 60 psi RAM pressure. The polymer was added first whereas MWNTs were added $30 \mathrm{~s}$ later. All mixes were removed from the internal mixer at a dump temperature of $145^{\circ} \mathrm{C}$ or lower with a total mix time of 3 to 5 minutes. Curatives of $1 \mathrm{phr}$ (parts per hundred of polymer) zinc oxide and $1.5 \mathrm{phr}$ stearic acid were subsequently added using a roller mill operating at $90^{\circ} \mathrm{C}$ 
to $100^{\circ} \mathrm{C}$. These blends were then compression molded and cured to form $2 \mathrm{~mm}$ thick plaques in a curing press. The cure temperature was set at $160^{\circ} \mathrm{C}$ and cure time applied was t90 +5 (5 minutes plus the time required to reach $90 \%$ cure as measured by an oscillating disk cure meter).

BIMSM/MWNT nanocomposites with a range in weight fraction of MWNT were produced. Thermogravimetric analysis (TGA) was conducted on all samples to verify the final material composition and found the prepared samples to possess a weight fraction close to that blended. Table 1 summarizes the compositions.

Scanning electron microscopy on freeze-fractured surfaces as well as transmission electron microscopy (TEM) (Figure 1) revealed good dispersion with random distribution and orientation of MWNTs within the elastomeric matrix.

\subsection{Mechanical Testing}

Uniaxial tension and uniaxial compression tests were conducted on all material compositions. Tests were conducted to large strains at constant engineering strain rate (displacement rate divided by specimen gage length) on a Zwick/Roell Z010 single axis screw machine. Specimens were cut from the $2 \mathrm{~mm}$ thick molded plaques. Tensile specimens were rectangular of dimension $60 \mathrm{~mm} * 5 \mathrm{~mm}$. Two types of grips were used. For moderate strains (strains less than 0.70) tensile grips with surfaces designed to test relatively thin compliant polymers were used; one surface is aluminum and the mating surface is a polyurethane. For larger strains (to final failure), Zwick grip 8122 with a spring-loaded grip action was used to avoid any sliding at very large deformation. The transverse displacement was monitored optically during extension and the axial strain was determined assuming incompressibility. Load-unload-reload tests were conducted to quantify any hysteresis and/or straininduced softening of the materials. Tests were also conducted at different engineering strain rates to quantify any rate dependence. Some samples were also extended until failure to examine the effect of MWNTs on the tensile stress and strain at break. Compression specimens were cylinders of nominally $8 \mathrm{~mm}$ in diameter and $6 \mathrm{~mm}$ in height (accomplished by stacking three $2 \mathrm{~mm}$ thick cylinders). Thin Teflon sheets were placed between the specimens and the compression platens to reduce friction; additionally, a powder composed of PTFE and PE was placed between the specimens and the Teflon sheets. Load-unload-reload tests were conducted. Tests were also performed at different engineering strain rates. Tensile and compression data are reported in terms of true stress (load divided by current deformed crosssectional area) and true strain (the natural logarithm of the current axial gage length divided by the initial gage length). Since the bulk modulus of rubber is substantially (i.e. 2-3 orders of magnitude greater) than the shear modulus of rubber, the deformed cross-sectional area during uniaxial tension and compression can be estimated from the measured axial strain assuming incompressibility. A minimum of 
at least three tests were conducted for each loading situation; stress-strain curves were repeatable in all cases and, in the case of uniaxial tension, strain-to-break measurements were also repeatable for all data sets.

\section{Experimental results}

\subsection{Uniaxial tension}

Uniaxial tension true stress-strain data are shown in Figure 2. Figure 2a reveals the elastomer matrix material to exhibit a small level of hysteresis (tests at different strain rates, not shown, revealed negligible strain rate sensitivity); the stiffness and the hysteresis are both observed to increase with an increase in MWNT content. The hysteresis was further quantified through cyclic load-unload-reload tests (representative data are provided in Appendix A) and was found to be a Mullins effect (see, Mullins (1947, 1969), Mullins and Tobin $(1954,1965)$ ) whereby the hysteresis was nearly absent after the first cycle. Figure $2 b$ depicts the uniaxial tensile data to large strain up to final failure where the stiffening with strain is observed to increase with increase in MWNT content and the strain to failure was also found to increase with increase in MWNT content (failure occurred in the gage length of the specimen and was not a grip-induced failure). The inset of Figure $2 \mathrm{~b}$ shows the initial modulus as a function of wt\% MWNT where the $12.2 \mathrm{wt} \%$ MWNT-elastomer composite has a modulus 2.5 times that of the neat elastomer.Comparatively, a similar weight fraction of carbon black filler would provide a modulus 1.3 times that of the neat elastomer (e.g., Bergstrom and Boyce (1999)). These data show an ability to substantially increase the tensile stiffness and retain the stiffness enhancement during large strain deformation as well as the ability to increase the tensile strength and the tensile strain-to-break.

\subsection{Uniaxial Compression Results}

Uniaxial compressive true stress-true strain data are shown in Figure 3a revealing the same basic trends as observed in the tensile data. The overall stiffness and the stiffening with strain are seen to increase with MWNT content. The neat elastomer exhibits a low level of hysteresis; the nanocomposite hysteresis increases with an increase in MWNT content. The compressive stress-strain behavior is compared to the tensile stress-strain behavior in Figure $3 \mathrm{~b}$ for the neat elastomer and for the $6.1 \mathrm{wt} \%$ and $12.2 \mathrm{wt} \%$ MWNT-elastomers. These data show the classic differences seen in elastomer compression vs tension stress-strain data. In an elastomer, this difference is attributable to the evolution in alignment of the underlying molecular

network with different states of strain. The network aligns in a biaxial orientation 
when subjected to uniaxial compression and aligns in a uniaxial orientation when subjected to uniaxial tension. Here, the MWNTs do not alter this compression vs tension dependence of the stress-strain behavior and, indeed, must also orient with deformation as discussed further in the modeling section.

\subsection{Strain Energy}

The tensile and compression stress vs strain data were further reduced to plots of strain energy density vs axial strain. Figure 4 shows the strain energy density results for all material compositions for uniaxial tension (Figure 4a) and uniaxial compression (Figure $4 \mathrm{~b}$ ). This data is then once again reduced to provide a plot of the strain energy contribution of the MWNTs, $U_{M W N T}$, to the composite behavior. Here, we assume a simple rule of mixtures approach giving:

$$
U_{c}=f U_{M W N T}+(1-f) U_{e}
$$

or,

$$
U_{M W N T}=\left(U_{c}-(1-f) U_{e}\right) /(f)
$$

where $U_{c}$ is the strain energy density of the composite, $U_{e}$ is the strain energy density of the elastomer, and $f$ is the volume fraction of the MWNTs. Figure 5 depicts $U_{M W N T}$ vs. axial strain for uniaxial tension (Figure 5a) and for uniaxial compression (Figure 5b) as obtained by reducing the data for all compositions. Note that the curves obtained from each composition are nearly coincident for the tensile data as well as for the compression data and thus reflect the unit contribution of the MWNTs to the deformation behavior of the composite. The coincidence of these data supports the use of equation 2 as a surprisingly good first approximation to assess and model the contribution of MWNTs to the composite behavior. This form of the reduced data will be shown to be instrumental in constructing the microstructurally-informed constitutive model for these nanocomposites ${ }^{1}$.

1 The loading curves were used to construct the strain energy density curves and, hence, contain the hysteresis contibutions seen in the elastomer compositions which were found to increase with an increase in MWNT content. As a first-order approach we are neglecting hysteresis effects. 


\section{Constitutive model}

\subsection{Model Development}

Our experimental results indicate that the strain energy density, $U_{c}$, of the MWNTelastomer composites under consideration can be decomposed into a contribution from the elastomer, $U_{e}$, and a contribution from the MWNTs, $U_{M W N T}$, using the simple rule of mixtures approach given earlier in equation (1). This representation is physically realistic for this case of a low to modest volume fraction of wavy (bent) fibers distributed within a compliant elastomeric matrix, where the fibers will essentially deform with the matrix, unbending and rotating with the imposed deformation. The wavy fibers offer little disturbance to the basic deformation field of the elastomer (in other words, there is not a significant amplification of strain in the matrix) and hence the elastomer deformation can be directly described by the macroscopic deformation gradient.

Here, the elastomer matrix contribution will be modeled using the Arruda-Boyce (A-B) eight-chain network model of rubber elasticity (Arruda and Boyce (1993)) and the MWNT contribution will be captured using the framework for anisotropic hyperelastic materials as originally developed by Spencer (1984). The strain energy contributions will be described below and each will be a function of properties of the constituent material and the imposed deformation. The macroscopic deformation is described by the deformation gradient, $\mathbf{F}=\partial \mathbf{x} / \partial \mathbf{X}$, with polar decompositiom $\mathbf{F}=\mathbf{R} \mathbf{U}=\mathbf{V} \mathbf{R}, \mathbf{X}$ denotes the position of a material point in the initial, reference configuration and $\mathbf{x}$ denotes the the position vector of that same material point in current, deformed configuration. The corresponding right Cauchy-Green tensor is given by $\mathbf{C}=\mathbf{F}^{\mathbf{T}} \mathbf{F}=\mathbf{U}^{2}$ and the left Cauchy-Green tensor is given by $\mathbf{B}=\mathbf{F F}^{\mathbf{T}}=\mathbf{V}^{\mathbf{2}}$. The invariants of $\mathbf{C}$ and $\mathbf{B}$ are given by:

$$
\begin{aligned}
& I_{1}=\operatorname{trace}(\mathbf{B})=\left(\lambda_{1}^{2}+\lambda_{2}^{2}+\lambda_{3}^{2}\right) \\
& I_{2}=\frac{1}{2}\left[I_{1}^{2}-\operatorname{trace}\left(\mathbf{B}^{2}\right)\right]=\lambda_{1}^{2} \lambda_{2}^{2}+\lambda_{1}^{2} \lambda_{3}^{2}+\lambda_{2}^{2} \lambda_{3}^{2} \\
& I_{3}=\operatorname{det}(\mathbf{B})=\lambda_{1}^{2} \lambda_{2}^{2} \lambda_{3}^{2}
\end{aligned}
$$

where $\lambda_{i}$ are the principal stretches.

\subsubsection{Elastomer Matrix Representation}

The BIMSM elastomer is a crosslinked molecular network and was found to exhibit little time-dependence. The Mullins effect (quantified in the data presented in Appendix A) is small and can be modelled using the Qi and Boyce (2004) model, 
but this effect is neglected in this paper in order to more clearly focus on the primary contributions of the MWNTs to the mechanical behavior. Hence, the matrix is approximated as hyperelastic and modeled using the A-B eight-chain network model of rubber elasticity. The A-B model represents a random isotropic molecular network as eight chains emanating from a central junction point to the corners of a cube; the cube is taken to be aligned with the principal axes of stretch. In this representation, each chain stretches by the root mean square of the imposed stretch, $\lambda_{\text {chain }}=\sqrt{\left(\lambda_{1}^{2}+\lambda_{2}^{2}+\lambda_{3}^{2}\right) / 3}=\sqrt{I_{1} / 3}$ and also rotates towards the axis(es) of maximum principal stretch(es) (Figure 6). Thus, the eight-chain model captures the essence of the physics of deformation of an isotropic random network as verified by its ability to predict the stress-strain behavior of an elastomer under different states of strain. The strain energy function for a compressible version of the eight-chain model (see, Bischoff et al. (2001)) is given by:

$$
U_{e}=\mu_{R}\left(\sqrt{N} \lambda_{\text {chain }} \beta+N \ln \frac{\beta}{\sinh \beta}-\frac{\sqrt{N} \beta_{o}}{3} \ln J\right)+\frac{1}{2} K_{B}(J-1)^{2}
$$

where $\beta=L^{-1}\left(\frac{\lambda_{\text {chain }}}{\sqrt{N}}\right) ; \lambda_{\text {chain }}=\sqrt{I_{1} / 3} ; \beta_{o}=L^{-1}\left(\frac{1}{\sqrt{N}}\right)$; where $L^{-1}$ is the inverse Langevin function with $L(\beta)=\operatorname{coth}(\beta)-1 / \beta, J=\sqrt{I_{3}}$ and $K_{B}$ is the bulk modulus.

The Cauchy stress is then obtained by differentiating $U_{e}$, giving:

$$
\mathbf{T}_{e}=\frac{2}{J} \frac{\partial U_{e}}{\partial I_{1}} \mathbf{B}+\frac{\partial U_{e}}{\partial J} \mathbf{1}
$$

or

$$
\mathbf{T}_{e}=\frac{\mu_{R}}{3 J} \sqrt{N}\left(\frac{\beta}{\lambda_{\text {chain }}} \mathbf{B}-\beta_{o} \mathbf{1}\right)+K_{B}(J-1) \mathbf{1}
$$

The material properties were fit to the BIMSM tensile data, giving $\mu_{R}=0.35 \mathrm{MPa}$ and $N=200$; the bulk modulus was taken to be $3 G P a$. The model fit is shown to capture the tensile behavior in Figure 7. As is well known, elastomeric stress-strain behavior is strongly dependent on the state of imposed deformation (see, for example, Treloar (1975), Arruda and Boyce (1993)), where the greatest contrast is seen in comparisons of uniaxial tension to uniaxial compression (or, alternatively, uniaxial tension and equibiaxial tension). The model prediction of the uniaxial compression behavior based on its fit to the tensile data is also shown in Figure 7 , demonstrating the ability of the model to capture the matrix behavior. The small discrepancy between model prediction and experiment is most likely due to our neglecting the small amount of hysteresis and Mullins effect (see Appendix A) exhibited by this material. However, for purposes of examining the effect of CNTs on the uniaxial tension and compression behavior, this discrepancy is small. 


\subsubsection{MWNT Representation}

The contribution to the composite strain energy emanating from the deformation of the constituent MWNTs is modeled by first building from the basic framework presented in Spencer (1984) for anisotropic fiber-reinforced hyperelastic materials. This framework has been followed and/or built upon in recent years by a number of investigators including, for example, Bischoff et al. (2002b,a) who established an anisotropic molecular network model for skin tissue; Holzapfel $(2003,2000)$ in developments of models of arterial wall tissue, and Reese et al. (2001) in models of fabric reinforced elastomers and Horgan and Saccomandi (2005) in a model of an incompressible elastomer reinforced with a single family of fibers. The framework begins with the recognition of fiber direction, a, as a building block of needed invariants for constructing the anisotropic strain energy function. Using a notation $\mathbf{a}_{\mathbf{i}}$ as representing the direction of the ith family of fibers, several additional invariants (beyond $I_{1}, I_{2}$, and $I_{3}$ introduced previously) become relevant. The primary invariants of interest being:

$$
\begin{aligned}
& I_{4_{i}}=\mathbf{a}_{i} \cdot \mathbf{C} \cdot \mathbf{a}_{i} \\
& I_{5_{i}}=\mathbf{a}_{i} \cdot \mathbf{C}^{2} \cdot \mathbf{a}_{i}
\end{aligned}
$$

where additional invariants which capture coupling between fiber families constitute the remaining invariants (for an overview, see, for example, Holzapfel (2000)).

In our representation, based on the experimental data, we first take the simplest approach and capture the influence of the wavy MWNTs (i.e., the MWNTs are not straight nor are they aligned, instead they are randomly dispersed and in an undulated or bent configuration within the matrix) by simply building a strain enregy function based on the $I_{4_{i}}$, where $I_{4_{i}}$ physically represents the square of the stretch of the fiber family in the ith direction: $\lambda_{M W N T_{i}}^{2}=I_{4_{i}}$. The MWNT strain energy contribution can then be expressed as:

$$
f U_{M W N T}=\sum_{i} f_{i} U_{M W N T}\left(\lambda_{M W N T_{i}}^{2}\right)
$$

where $f_{i}$ is the volume fraction of MWNTs whose end-to-end vector is aligned in the ith direction and the sum is over all MWNT end-to-end directions present in the material; the "fiber stretch" here refers to the extension of the end-to-end distance of this wavy MWNT where this extension results in the unbending or straightening out of the MWNT waviness.

For the material under consideration, we note that the orientation distribution is isotropic and we thus take a leap similar to that of the eight-chain network model of rubber elasticity: we represent the contribution of the random distribution of nanotubes by the average initial orientation direction. The average orientation is approximately given by the azimuthal angle of 55 degrees (see, for example, Bergstrom and Boyce (2001)) corresponding to the fibers associated with the $\langle 1,1,1\rangle$ family 
(i.e. $(1,1,1),(1,1,-1),(-1,1,1),(1,-1,1) \ldots)$. This isotropic distribution is more optimally captured by considering the average orientation given with respect to the principal stretch directions, i.e. taking the azimuthal angle of $55^{\circ}$ with respect to the maximum principal stretch direction. This is thus the "8-chain" network approach and gives: $\lambda_{M W N T}=\sqrt{I_{1} / 3}$. We thus have "isotropized" the anisotropic strain energy function for a random isotropic distribution of MWNTs which gives the MWNT contribution to be:

$$
f U_{M W N T}=f \hat{U}_{M W N T}\left(\lambda_{M W N T}^{2}\right)
$$

where $\lambda_{M W N T}^{2}=I_{1} / 3$.

This isotropized contribution also provides a framework for further reduction of our experimental data of Figures 5a and 5b which we can now represent as $U_{M W N T}$ vs $\left(\lambda_{M W N T}^{2}-1\right)$ as shown in Figure 8. Fitting the data as represented in Figure 8 provides the MWNT strain energy function:

$$
U_{M W N T}=A_{1}\left(\lambda_{M W N T}^{2}-1\right)+A_{2}\left(\lambda_{M W M T}^{2}-1\right)^{2}-\frac{2 A_{1}}{3} \ln J
$$

or,

$$
U_{M W N T}=\frac{A_{1}}{3}\left(I_{1}-3\right)+\frac{A_{2}}{9}\left(I_{1}-3\right)^{2}-\frac{2 A_{1}}{3} \ln J
$$

where the model curve fit, with $A_{1}=6.68 \mathrm{MPa}$ and $A_{2}=2.29 \mathrm{MPa}$, is shown in Figure 9. The model for the more general case of a distribution in MWNT orientations is given in Appendix B. We further emphasize that the strain energy contribution from the MWNTs is a result of the energy from bending and/or unbending of undulations in the MWNTs during the imposed macroscopic deformation, where the MWNTs stretch (extending their end-to-end distance via unbending) and rotate with the imposed deformation such that they deform with the matrix.

\subsubsection{Composite Constitutive Model}

The composite strain energy function is simply the sum of the contributions from the elastomer and from the MWNT as given earlier in equation 1 and now using equations 4 and 9 for the elastomer and MWNT contributions, respectively. Differentiation of the strain energy function gives the Cauchy stress:

$$
\begin{aligned}
\mathbf{T} & =(1-f) \frac{\mu_{R}}{3 J} \sqrt{N}\left(\frac{\beta}{\lambda_{\text {chain }}} \mathbf{B}-\beta_{o} \mathbf{1}\right) \\
& +\frac{2}{3} f\left(A_{1}+2 \frac{A_{2}}{3}\left(I_{1}-3\right)\right) \mathbf{B}-\frac{2 f A_{1}}{3} \mathbf{1}+K_{B}(J-1) \mathbf{1}
\end{aligned}
$$


The constitutive model results are compared to data in Figures 10 and 11. Figure 10 shows the model results for uniaxial tension of the neat elastomer and the MWNT-elastomer composites plotted together with the data. The model is found to quantitatively capture the features of the uniaxial tension stress-strain behavior and its dependence on MWNT content. The inset of Figure 10 shows the model results for initial modulus as a function of wt $\%$ MWNT. The model results for uniaxial tension and compression to strains of 1.0 and -1.0 , respectively, together with the corresponding data are shown in Figure 11. The model is also found to be in good agreement with experimental data for uniaxial compression. The model is found to capture the data to large strain and to quantitatively predict the uniaxial compression behavior showing the distinct differences in tension and compression data as well as the dependence of the curves on MWNT content.

\section{Conclusions}

The influence of multi-wall carbon nanotubes on the mechanical behavior of elastomeric materials was investigated. The BIMSM elastomer was blended with multiwall carbon nanotubes (MWNTs) and the influence of weight fraction of MWNTs on the large deformation tensile and compressive behavior was quantifed. The initial stiffness and subsequent stretch-induced stiffening at large strains were both found to increase with MWNT content. The MWNTs were also found to increase the tensile strength and the tensile strain-to-break. The strain energy density of the composite materials as obtained experimentally was reduced into two contributions: a matrix contribution and a MWNT contribution. The ability to decompose the experimentally obtained strain energy guided the development of a hyperelastic constitutive model for the composite materials based on the volume fraction of matrix and MWNT. The model was found to predict the effects of MWNT content on the large deformation stress strain behavior of the composite materials in tension and compression, showing excellent agreement with the experimental data.

\section{Acknowledgements}

This research was sponsored, in part, by USAFOSR through the DURINT Polymer Nanocomposites, Grant No. F49620-01-1-0447, and, in part, by ExxonMobil Chemical Company. 


\section{References}

Ahir, S., Squires, A., Rajbakhsh, A., Terentjev, E., 2006. Infrared actuation in aligned polymer-nanotube composites. Phys. Rev. B 73, 1-12.

Ahir, S., Terentjev, E., 2005. Photomechanical actuation in polymer nanotube composites. Nature Mater. 4, 491-495.

Arruda, E. M., Boyce, M. C., 1993. A three-dimensional constitutive model for the large stretch behavior of rubber elastic materials. Jnl. of the Mechanics and Physics of Solids 41, 389-412.

Baughman, R., Zakhidov, A., de Heer, W., 2002. Carbon nanotubes the route toward applications. Science 297 (5582), 787-792.

Bergstrom, J., Boyce, M., 1999. Mechanical behavior of particle filled elastomers. Rubber Chemistry and Technology 72, 633-656.

Bergstrom, J., Boyce, M., 2000. Large strain time-dependent behavior of filled elastomers. Mechanics of Materials 32, 627-644.

Bergstrom, J. S., Boyce, M. C., 2001. Deformation of elastomeric networks: relation between molecular level deformation and classical statistical mechanics models of rubber elasticity. Macromolecules 34, 614-626.

Bischoff, J. E., Arruda, E. M., Grosh, K., 2001. A new constitutive model for the compressibility of elastomers at finite deformation. Rubber Chemistry and Technology 74, 541-559.

Bischoff, J. E., Arruda, E. M., Grosh, K., 2002a. A microstructurally based orthotropic hyperelastic constitutive law. Jnl. Appl. Mech. 69, 570-579.

Bischoff, J. E., Arruda, E. M., Grosh, K., 2002b. Orthotropic hyperelasticity in terms of an arbitrary molecular chain model. Jnl. Appl. Mech. 69, 198-201.

Bradshaw, R., Fisher, F., Brinson, L., 2003. Fiber waviness in nanotube-reinforced polymer compositesii modeling via numerical approximation of the dilute strain concentration tensor. Comp. Sci. Technol. 63, 1705-1722.

Chen, W., Tao, X., Liu, Y., 2006. Carbon nanotube-reinforced polyurethane composite fibers. Comp. Sci. Technol.In press, available on line June 2006.

Chen, Y., Shaw, D., Guo, L., 2000. Field emission of different oriented carbon nanotubes. Applied Physics Letters 76 (17), 2469-2471.

Coleman, J. N., Khan U., B. W. J., Gunko, Y. K., 2006. Small but strong: A review of the mechanical properties of carbon nanotube polymer composites. Carbon 44, 1624-1652.

Cooper, C., Ravich, D., Lips, D., Mayer, J., Wagner, H., 2002. Distribution and alignment of carbon nanotubes and nanofibrils in a polymer matrix. Comp Sci Technol 62, 1105-1102.

Dalmas, F., Chazeau, L., Gauthier, C., Masenelli-Varlot, K., Dendievel, R., Cavaille, J., 2005. Multiwalled carbon nanotube/polymer nanocomposites: processing and properties. J. Polym. Sci. Part B: Polym Phys 43 (10), 1187-1197.

Dannenberg, E. M., 1975. The effet of surface chemical interactions on the properties of filler reinforced rubbers. Rubber Chemistry and Technology 44, 440-478.

Dufresne, A., Paillet, M., Putaux, J., Canet, R., Carmona, F., Delhaes, P., 2002. Processing and characterization of carbon nanotube/poly(styren-co-butyl acry- 
late) nanocomposites. Jnl. Mater. sci. 37, 3915-3923.

Frogley, M., D., R., H.D., W., 2002. Mechanical properties of carbon nanoparticlereinforced elastomers. Comp Sci Technol 63, 1647-1654.

Guth, E., 1945. Theory of filler reinforcement. Jnl. Appl. Phys. 16 (1), 20-25.

Harwood, J., Payne, A., 1966. Stress-softening in natural rubber vulcanizates. part iii: Carbon black-filled vulcanizates. J. Appl. Polym. Sci. 10, 315-324.

Holzapfel, G. A., 2000. Nonlinear solid mechanics, a continuum approach for engineering. John Wiley and Sons, Chichester.

Holzapfel, G. A., 2003. Structural and numerical models for the viscoelastic response of arterial walls with residual stresses. Springer-Verlag, Wien, chapter (pages 109-184) in Biomechanics of Soft Tissue in Cardivascular Systems, ed. Holzapfel, G.A., Ogden, R.W., CISM Lectures No. 441.

Horgan, C. O., Saccomandi, G., 2005. A new consititutive theory for fiberreinforced incompressible nonlinearly elastic solids. Jnl. of the Mechanics and Physics of Solids 53, 1985-2015.

Kilian, H. G. a., 1994. Universal properties in filler loaded rubbers. Rubber Chemistry and Technology 67.

Koerner, H., Liu, W., Alexander, M., Mirau, P., Dowty, H., Vaia, R., 2005. Deformation-morphology correlations in electrically conductive carbon nanotube-thermoplastic polyurethane nanocomposites. Polymer 46, 4405-4420.

Kresge, E. N., Wang, H. C., 1993. Butyl Rubbers. John Wiley and Sons, New york, v. 8 p 934 in Kirk-Ohmer Encyclopedia of Chemical Technology.

Mullins, L., 1947. Effect of stretching on the properties of rubber. Journal of Rubber Research 16, 275-289.

Mullins, L., 1969. Softening of rubber by deformation. Rubber Chem. Technol. 42, 339-362.

Mullins, L., Tobin, N. R., 1954. Theoretical model for the elastic behavior of fillerreinforced vulcanized rubbers. Proc. 3rd Rubber Technol. Con., W. Heffer and Sons Ltd., 397-412.

Mullins, L., Tobin, N. R., 1965. Stress softening in ruber vulcanizates. parti. use of a strain amplification factor describe the elastic behavior of filler-reinforced vulcanized rubber. Jnl. of Applied Polymer Science 9, 2993-3009.

Powers, K. W., Wang, H. C., Chung, T. C., Dias, A. J., Olkusz, J. A., November 10 1992. Patent : Para-alkylstyrene/isolefin copolymers and functionalized copolymer thereof. Number : US 5,162,445.

Qi, H. J., Boyce, M. C., 2004. Constitutive model for stretch-induced softening of the stress-stretch behavior of elastomeric materials. Jnl. of the Mechanics and Physics of Solids 52, 2187-2205.

Qian, D., Dickey, E., Andrews, R.and Rantell, T., 2000. Load transfer and deformation mechanisms in carbon nanotubepolystyrene composites. Adv. Mater. 76 (20), 2868-2870.

Reese, S., Raible, T., Wriggers, P., 2001. Finite element modelling of orthotropic material behavior in pneumatic membranes. Int. Jnl. of Solids and Structures 38, 9525-9544.

Safadi, B., Andrews, R., Grulke, E., 2002. Multiwalled carbon nanotube polymer 
composites: synthesis and characterization of thin films. Jnl. of Applied Polymer Science 84 (14), 2660-2669.

Shaffer, M., Windle, A., 1999. Fabrication and characterization of carbon nanotube/poly(vinyl alcohol) composites. Adv. Mater. 11, 937-941.

Spencer, A. J. M., 1984. Constitutive Theory for strongly Anisotropic Solids. Springer-Verlag, Wien, chapter (pages 1-32) in Continuum theory of the mechanics of fiber-reinforced composites, ed. Spencer, A. J. M., CISM Courses and Lectures No. 282.

Treloar, L., 1975. The Physics of Rubber Elasticity. 3rd ed., Clarendon Press, Oxford.

Wong, E., Sheehan, P., C.M., L., 1997. Nanobeam mechanics: elasticity, strength, and toughness of nanorods and nanotubes. Science 277, 1971-1975. 
Table 1: Material Compositions

\begin{tabular}{|c||c|c|c|c|}
\hline Nanocomposite No. & 1 & 2 & 3 & 4 \\
\hline \hline Elastomer $(\mathrm{phr})$ & 100 & 100 & 100 & 100 \\
\hline MWNT $(\mathrm{phr})$ & 2.5 & 7.5 & 10 & 15 \\
\hline Formulation $(W / f)$ & $2.43 / .0113$ & $6.97 / .0372$ & $9.09 / .049$ & $13.04 / .0718$ \\
\hline TGA $(W / f)$ & $2.60 / .0136$ & $6.07 / .0323$ & $8.8 / .0474$ & $12.16 / .0667$ \\
\hline
\end{tabular}

$W=$ weight percent; $f=$ volume fraction
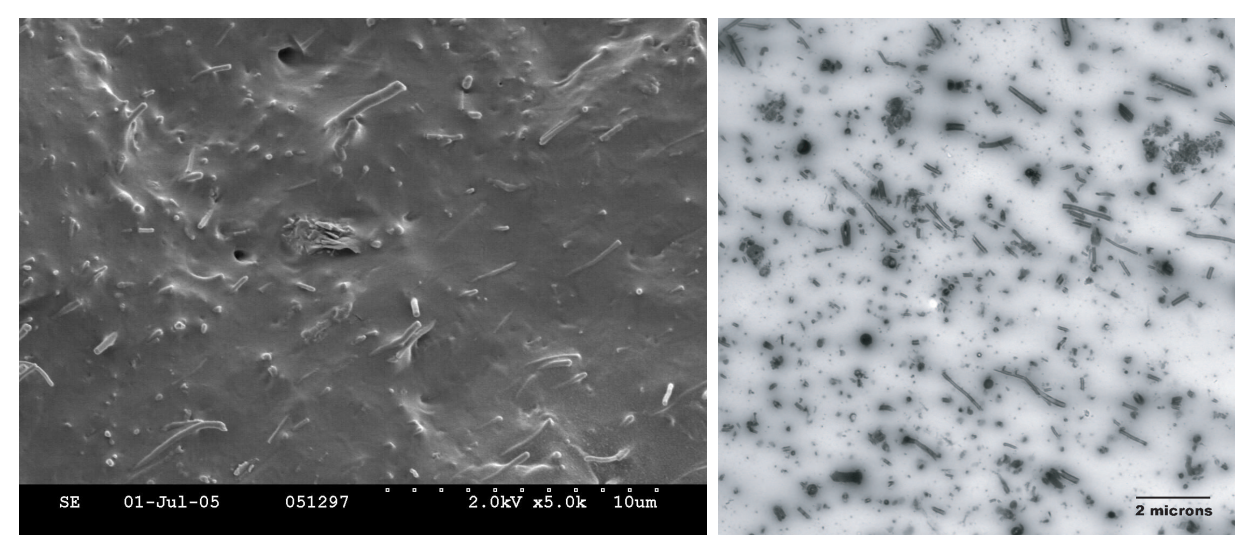

Fig. 1. (a) Low magnification Scanning Electron micrographs of a freeze fractured surface and (b) TEM of MWCNT/elastomer nanocomposite of $15 \mathrm{wt} \%$, showing uniformity of MWCNT dispersion 

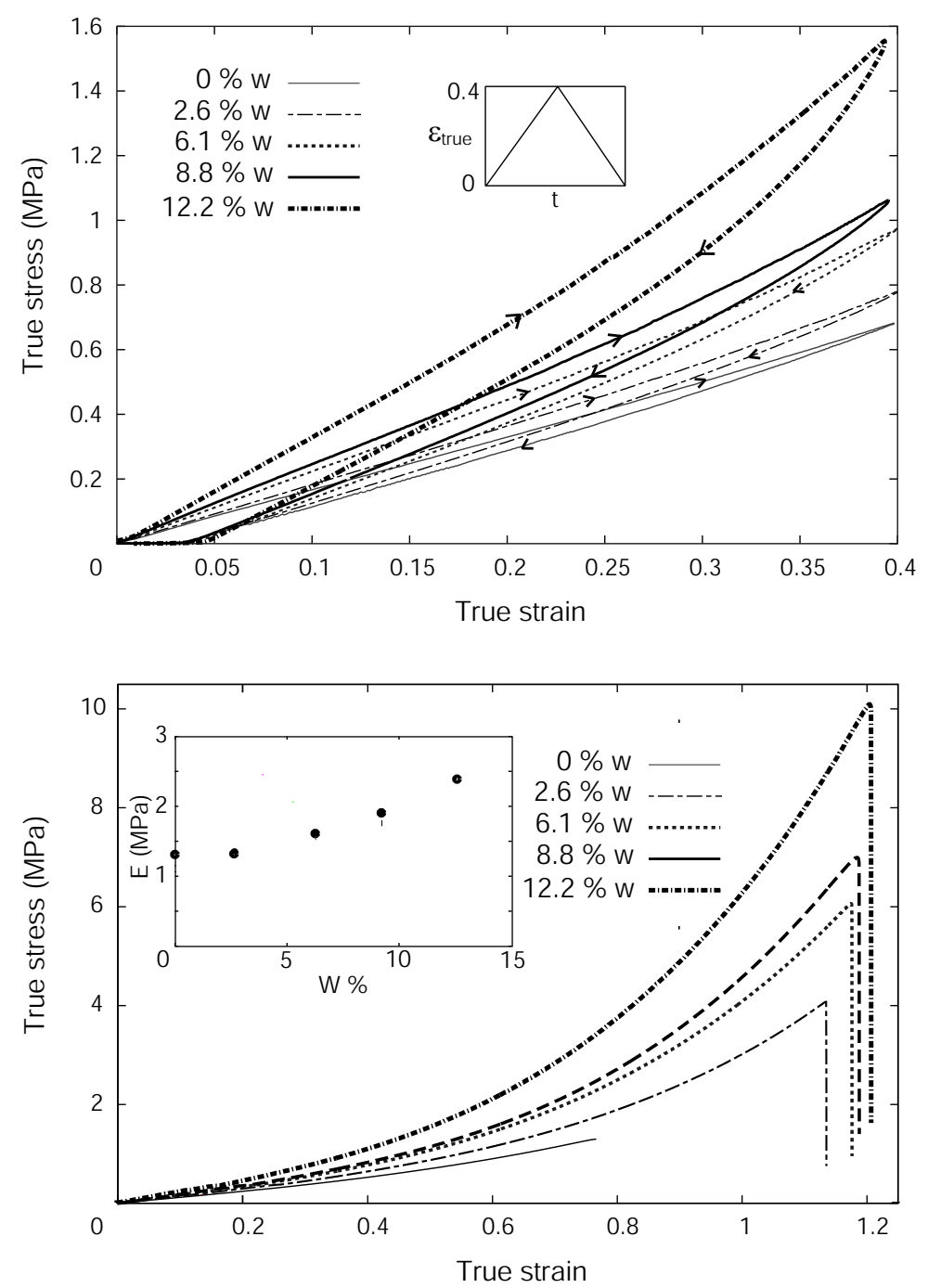

Fig. 2. (a) Uniaxial tension load-unload true stress vs. true strain at an engineering strain rate of $0.01 / \mathrm{s}$ to a strain of 0.4 for the elastomer and the elastomer-MWNT nanocomposites; (b)Uniaxial tension true stress vs. true strain at a strain rate of 0.01/s taken to failure; the inset plot reports the initial axial modulus as a function of weight fraction MWNT. 

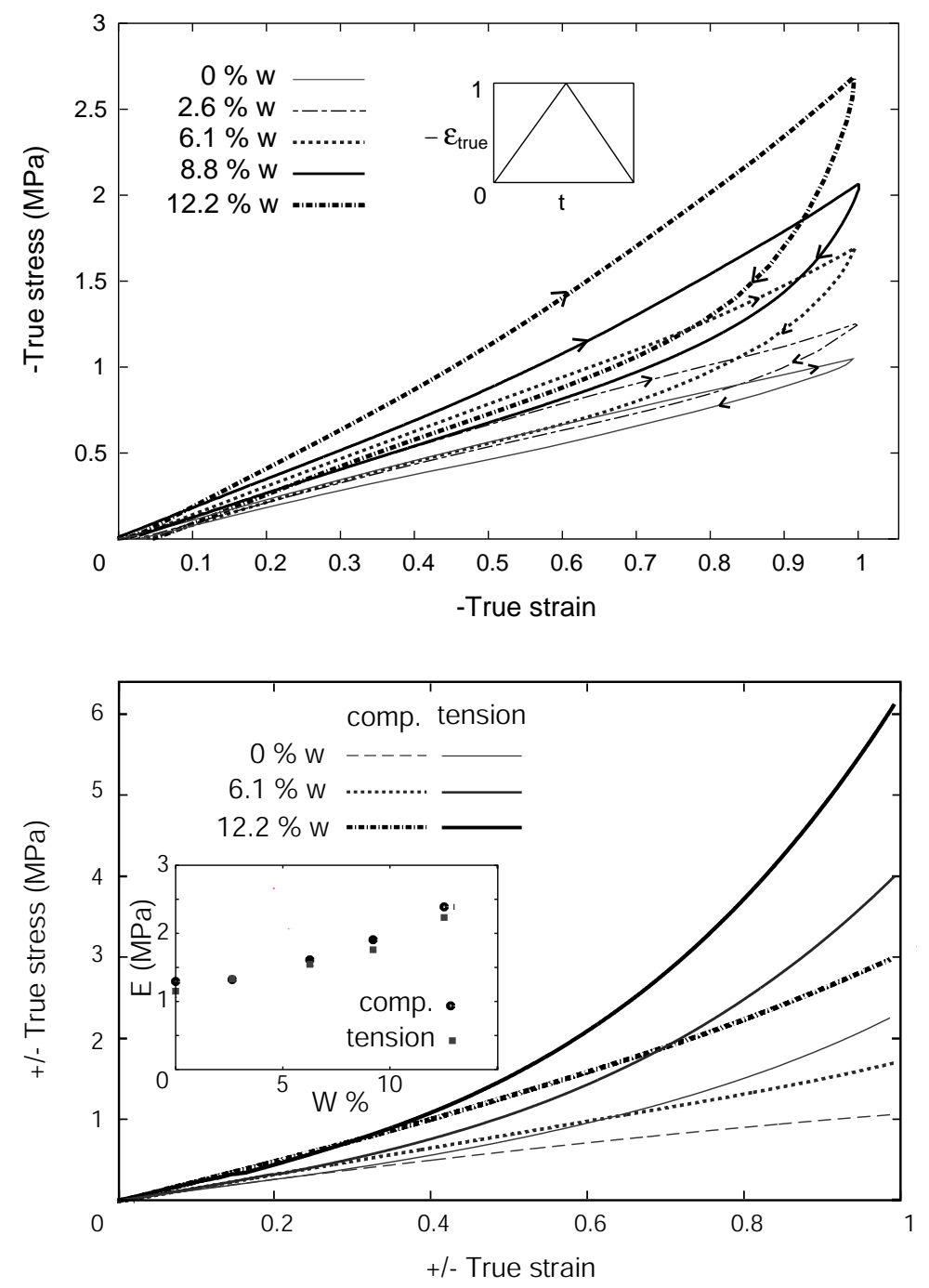

Fig. 3. (a) Uniaxial compression load-unload true stress vs. true strain at an engineering strain rate of 0.01/s to a strain of -1.0 for the elastomer and the elastomer-MWNT nanocomposites; (b) Comparison of uniaxial tension and uniaxial compression true stress-true strain for Ophr, $5 \mathrm{phr}(6.07 \% \mathrm{wt})$ and $15 \mathrm{phr}(12.16 \% \mathrm{wt})$ compositions; the inset plot reports the comparison of uniaxial tension and uniaxial compression initial modulus as a function of weight fraction MWNT. 

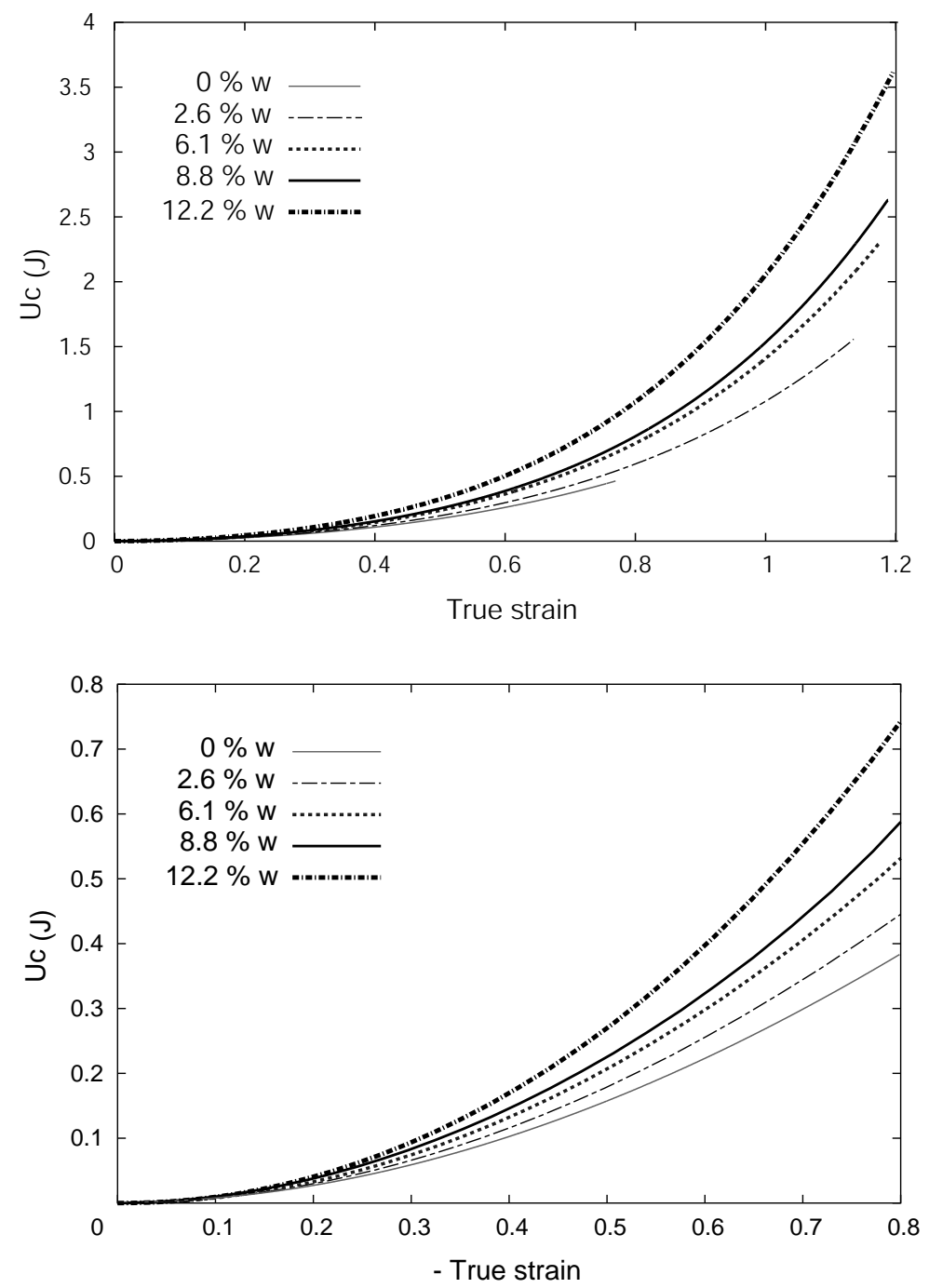

Fig. 4. Strain energy density vs true axial strain for all compositions for (a) uniaxial tension, and (b) uniaxial compression 

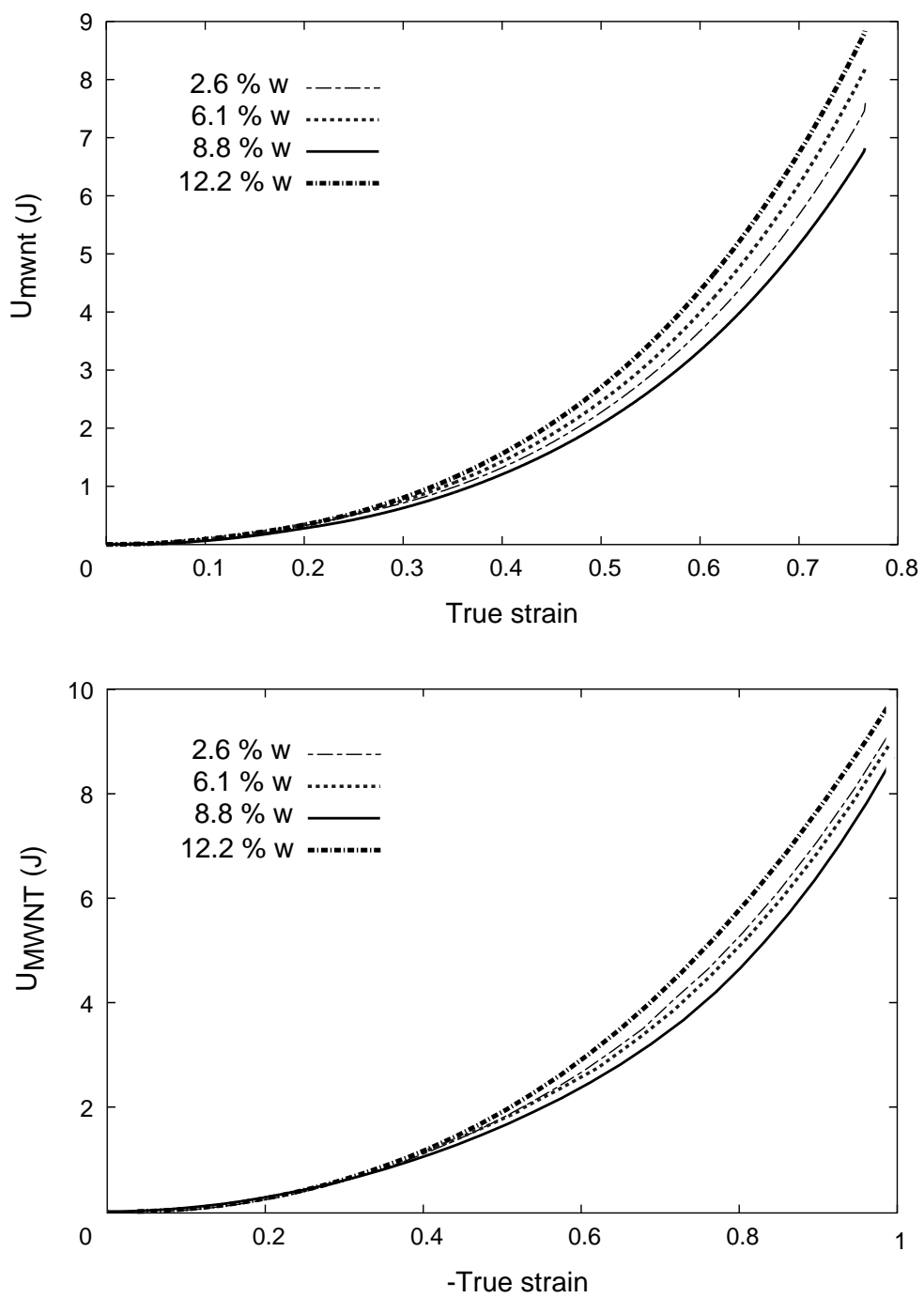

Fig. 5. Strain energy density of the MWNTs vs true axial strain for all compositions for (a) uniaxial tension and (b) uniaxial compression.
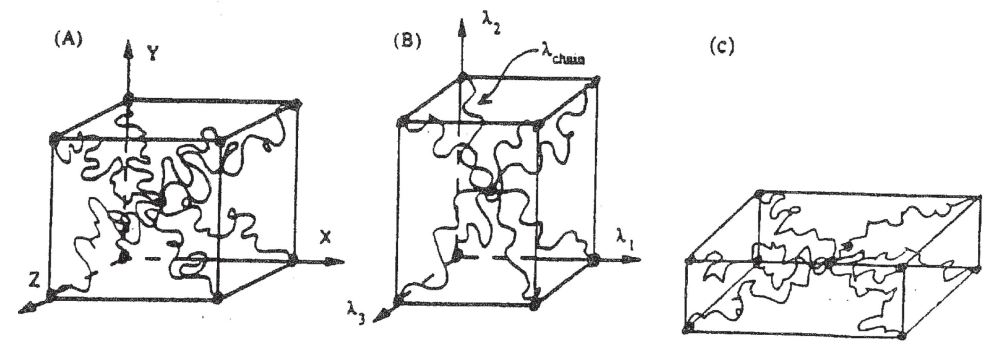

Fig. 6. Eight-chain network model is (a) undeformed, (b) uniaxial tension, and (c) uniaxial compression, Arruda and Boyce (1993) 


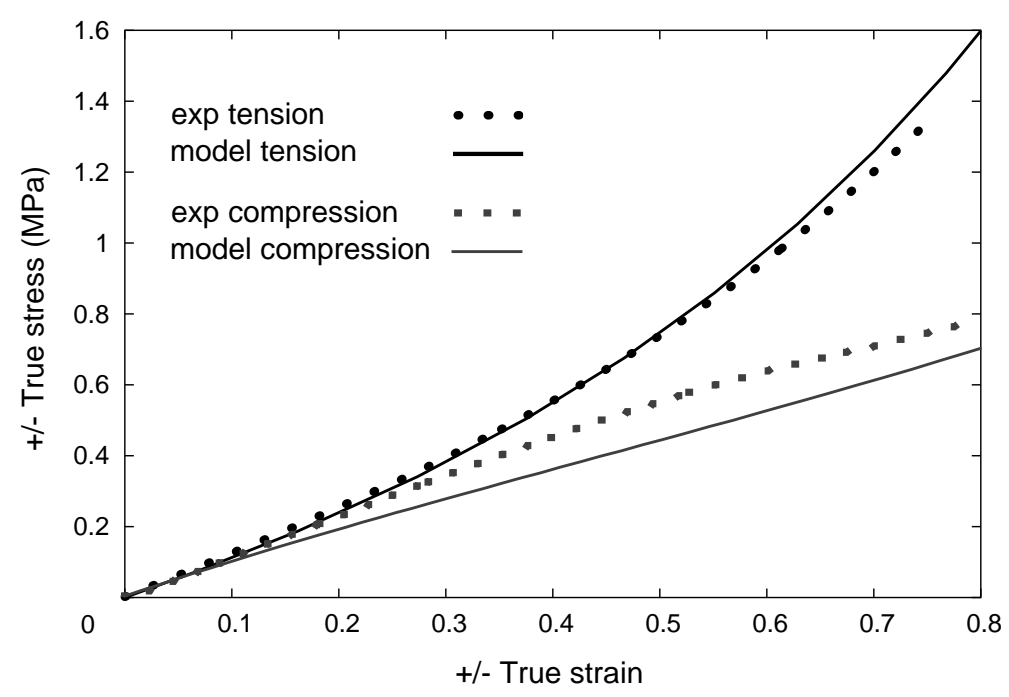

Fig. 7. Arruda Boyce model and experimental data for BIMSM in uniaxial tension and uniaxial compression. Model fit to tensile data; compression is a prediction.

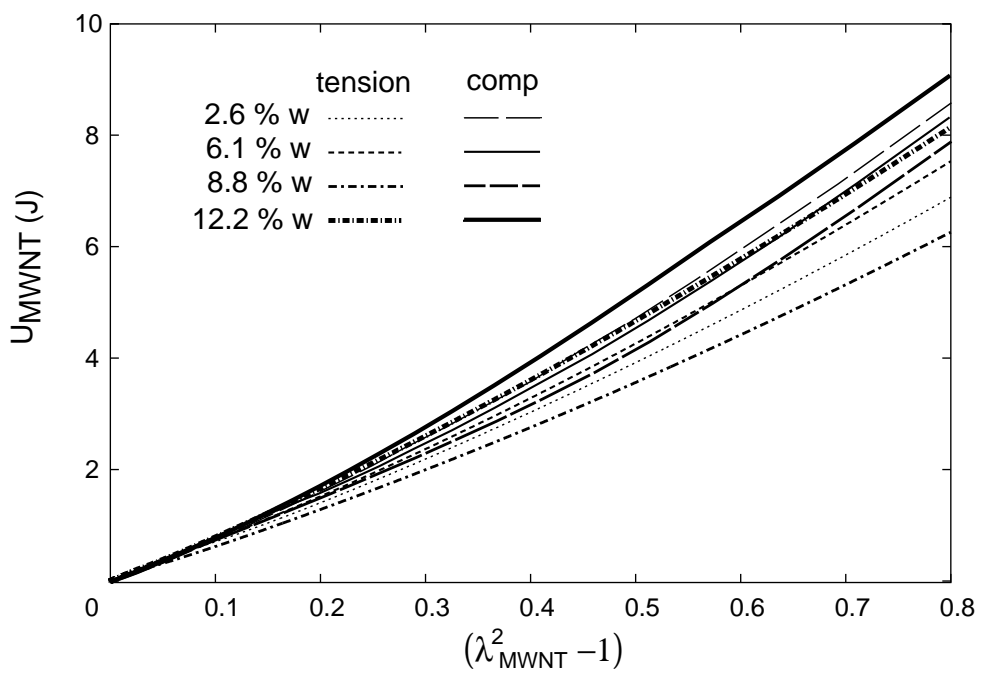

Fig. 8. $U_{M W N T}$ vs $\left(\lambda_{M W N T}^{2}-1\right)$ for uniaxial tension and for uniaxial compression. 


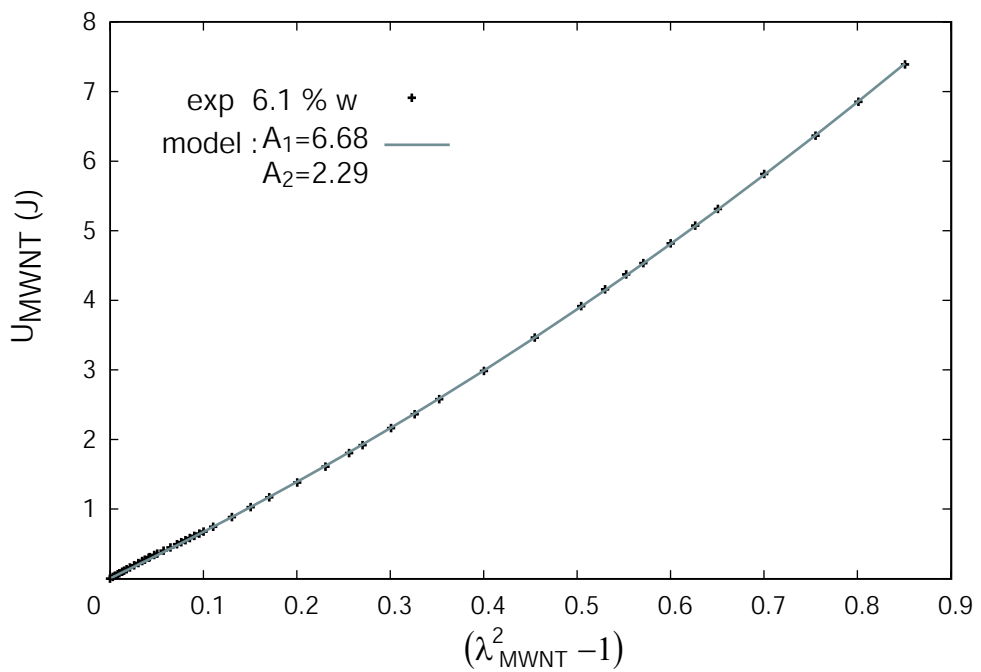

Fig. 9. Model and experimental data for $M W N T$ weight percents $6.1 \% W, U_{M W N T}$ vs $\left(\lambda_{M W N T}^{2}-1\right)$.

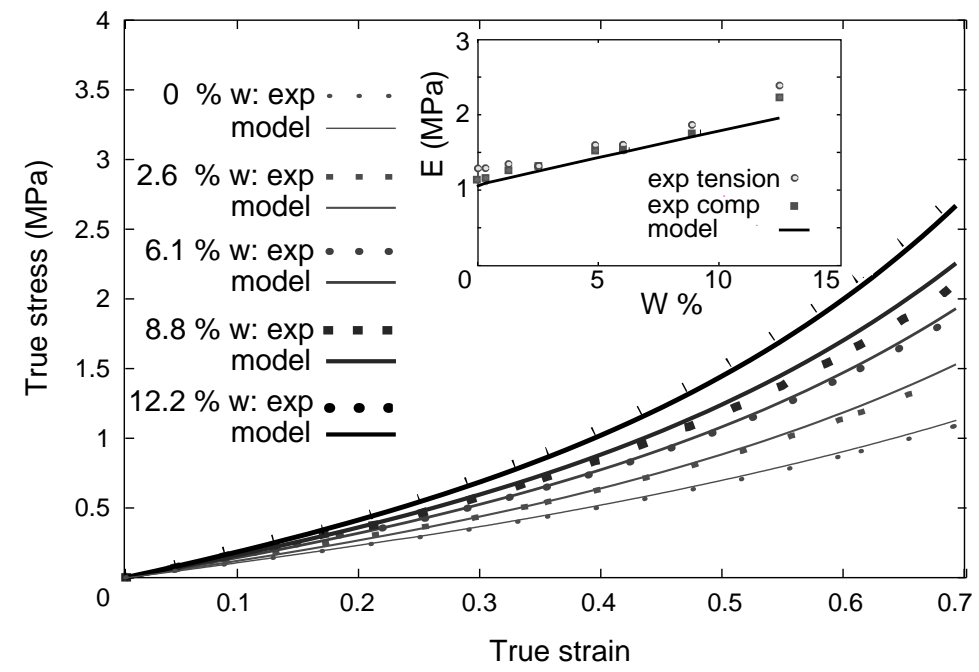

Fig. 10. Constitutive model results for uniaxial tension of MWNT-elastomer composites compared to data.; the inset plot reports the initial axial modulus as a function of weight fraction MWNT for model and experimental data . 

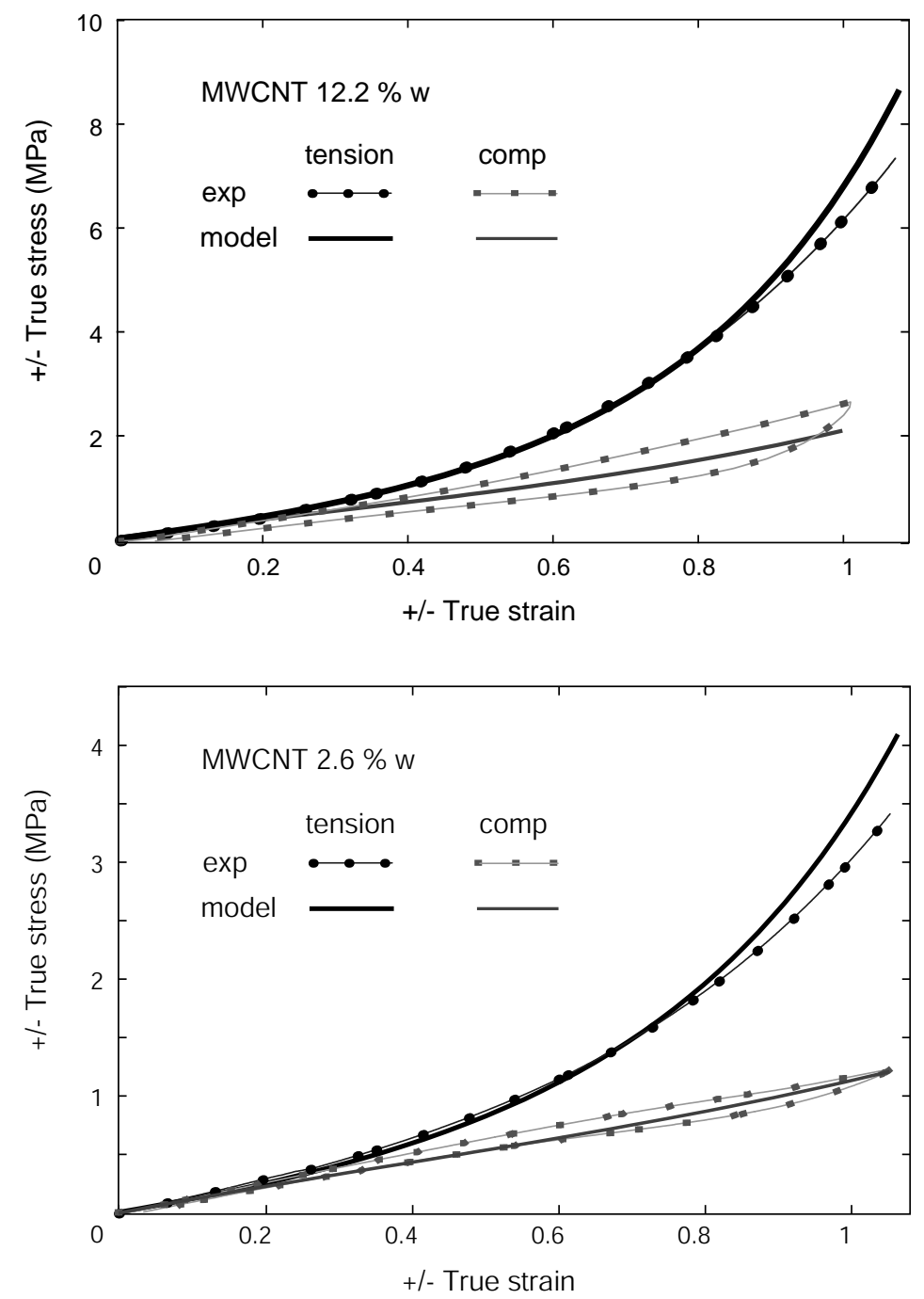

Fig. 11. Constitutive model results for uniaxial tension and uniaxial compression compared to data for MWNT weight percents (a) $12.2 \%$ and (b) $2.6 \%$. 


\section{A Mullins effect}

The elastomer and MWNT-elastomer composites of this study were found to exhibit a Mullins effect (a stretch-induced softening of the stress-strain behavior commonly observed in elastomeric material, see, for example, Mullins (1947, 1969), Mullins and Tobin (1954, 1965), Harwood and Payne (1966)) as evidenced by a more compliant response on reloading of the material after having been subjected to a prior load-unload cycle. For completeness, Figures A.1 and A.2 show loadunload-reload data for uniaxial tension (A.1) and compression (A.2) of the neat elastomer and MWNT-elastomers. Figure (A.1) shows the uniaxial tensile loadunload-reload behavior to/from strains of 0.40 and 0.69 for the neat elastomer and the 15phr MWNT-elastomer composite. Figure A.2 shows the load-unload-reload behavior of the neat elastomer and the $15 \mathrm{phr}$ MWNT-elastomer nanocomposite in compression to/from a strain of -1.0 .

\section{B Anisotropic Strain Energy for Composite Containing Initial Preferential Orientation of MWNTs}

The materials under consideration were found to contain a randomly oriented distribution of MWNTs. However, the modeling presented is also applicable to the case of an initially preferentially oriented distribution of MWNTs as is presented in this appendix for purposes of both completeness and comparison. The fibers direction are given by unit vectors $\mathbf{a}_{i}=x_{i} \mathbf{e}_{1}+y_{i} \mathbf{e}_{2}+z_{i} \mathbf{e}_{3}$ in the initial configuration and $\lambda_{I}=\sqrt{\mathbf{e}_{1} \cdot \mathbf{C} . \mathbf{e}_{1}}, \lambda_{I I}=\sqrt{\mathbf{e}_{2} \cdot \mathbf{C} . \mathbf{e}_{2}}, \lambda_{I I I}=\sqrt{\mathbf{e}_{3} . \mathbf{C . \mathbf { e } _ { 3 }}}$ represent the stretches along the principales fiber axes. We take the MWNT contribution to be given by:

$$
U_{M W N T}=\sum_{i} f_{M W N T_{i}} U_{M W N T}\left(\lambda_{M W N T_{i}}^{2}\right)
$$

where $\lambda_{M W N T_{i}}{ }^{2}=I_{4 i}=\mathbf{a}_{i} \mathbf{C} \mathbf{a}_{i}$ giving the composite strain energy function:

$$
U_{M W N T}=A_{1}\left(\lambda_{M W N T_{i}}{ }^{2}-1\right)+A_{2}\left(\lambda_{M W N T_{i}}{ }^{2}-1\right)^{2}-2 A_{1} \ln \left(\lambda_{I}^{x_{i}^{2}} \lambda_{I I}^{y_{i}^{2}} \lambda_{I I I}^{z_{i}^{2}}\right)
$$



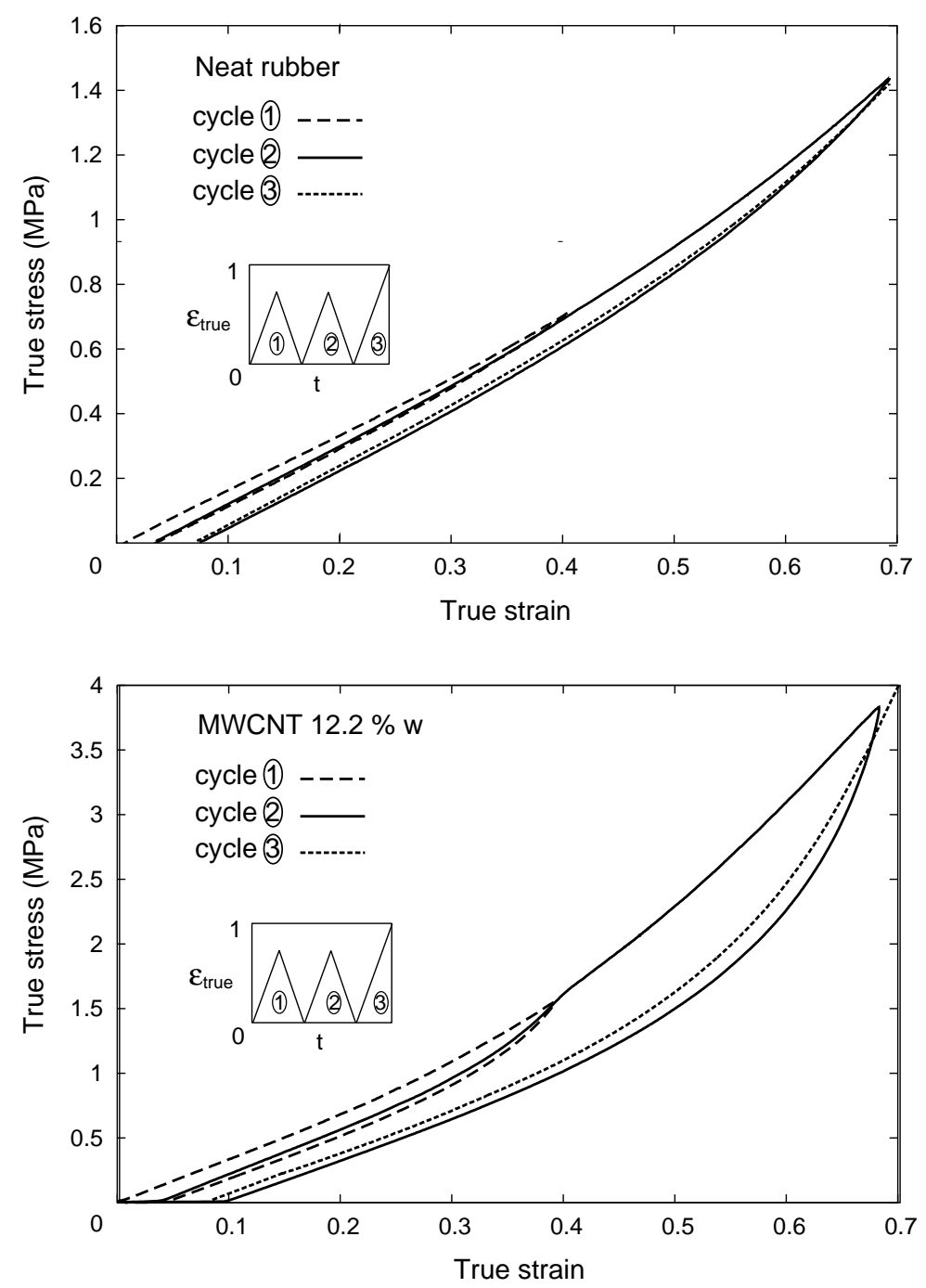

Fig. A.1.

Differentiation of strain energy function gives:

$$
\begin{aligned}
\mathbf{T} & =J^{-1} \mathbf{F}\left(2 \frac{\partial U_{e}}{\partial \mathbf{C}}+2 \frac{\partial U_{M W N T}}{\partial \mathbf{C}}\right) \mathbf{F}^{T} \\
& =\left(1-\sum_{i} f_{M W N T_{i}}\right) \frac{\mu_{R}}{3 J} \sqrt{N}\left(\frac{\beta}{\lambda_{\text {chain }}} \mathbf{B}-\beta_{o} \mathbf{1}\right) \\
& +\sum_{i} f_{M W N T_{i}} \lambda_{M W N T_{i}}{ }^{2}\left(2 A_{1}+4 A_{2}\left(\lambda_{M W N T_{i}}{ }^{2}-1\right)\right) \mathbf{a}_{i}^{\prime} \otimes \mathbf{a}_{i}^{\prime} \\
& -\frac{2 A_{1}}{J} \sum_{i} f_{M W N T_{i}} \mathbf{F}\left(\frac{x_{i}^{2}}{\lambda_{I}^{2}} \mathbf{e}_{1} \otimes \mathbf{e}_{1}+\frac{y_{i}^{2}}{\lambda_{I I}^{2}} \mathbf{e}_{2} \otimes \mathbf{e}_{2}+\frac{z_{i}^{2}}{\lambda_{I I I}^{2}} \mathbf{e}_{3} \otimes \mathbf{e}_{3}\right) \mathbf{F}^{T} \\
& +K_{B}(J-1) \mathbf{1}
\end{aligned}
$$

with $\mathbf{a}_{i}^{\prime}=\mathbf{F} . \mathbf{a}_{i}$ 


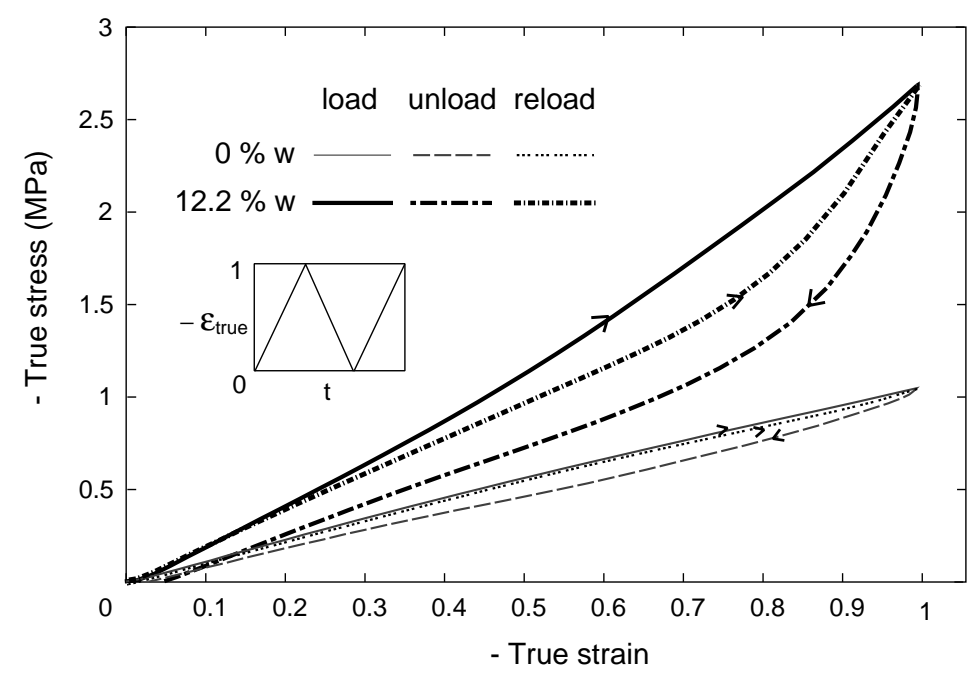

Fig. A.2.

Table B.1: Orientation Distribution for Each Case

\begin{tabular}{|c||c|c|c|}
\hline case No. & $\theta$ & $f_{i}$ & $a_{i}$ \\
\hline \hline 1 & 40 & 0.01667 & $(1,1,1.685),(-1,1,1.685),(1,-1,1.685),(-1,-1,1.685)$ \\
\hline 2 & 55 & 0.01667 & $(1,1,1),(-1,1,1),(1,-1,1),(-1,-1,1)$ \\
\hline 3 & 70 & 0.01667 & $(1,1,0.53),(-1,1,0.53),(1,-1,0.53),(-1,-1,0.53)$ \\
\hline 4 & 40,70 & 0.008335 & $(1,1,1.685),(-1,1,1.685),(1,-1,1.685),(-1,-1,1.685)$ \\
\hline & & 0.008335 & $(1,1,0.53),(-1,1,0.53),(1,-1,0.53),(-1,-1,0.53)$ \\
\hline
\end{tabular}

As an example, we compare four cases, each with a total volume fraction of MWNTs of $f=0.068$. The orientation distributions are represented as "cones" of fiber families (Figure B.1a) to the axial loading direction. Furthermore, to adequately capture each azimuthal angle family, four particular directions from each cone are taken as indicated in the figure. The four cases that we compare are listed in Table B.1. Results are presented for uniaxial tension and uniaxial compression of the four cases where Figure B.1a shows the uniaxial tensile behavior and Figure B.1b shows the uniaxial compression behavior. In tension and compression, the initial stiffness depends strongly on the initial alignment of the fibers (Figure B.2) . In tension simulations, the nanocomposite is observed to stiffen with strain and the strain stiffening is observed to increase with the initial fiber alignment, where nanocomposites with fibers initially more axially oriented stiffen more with strain. In compression simulations, the strain stiffening is also found to depend upon initial fiber orientation, where initial alignments with the fibers more biaxially oriented (in the plane with normal coinciding with the compression axis) are observed to strain stiffen more with strain as the fibers become more biaxially aligned in the plane normal to the compression axis thus resisting the compression. 

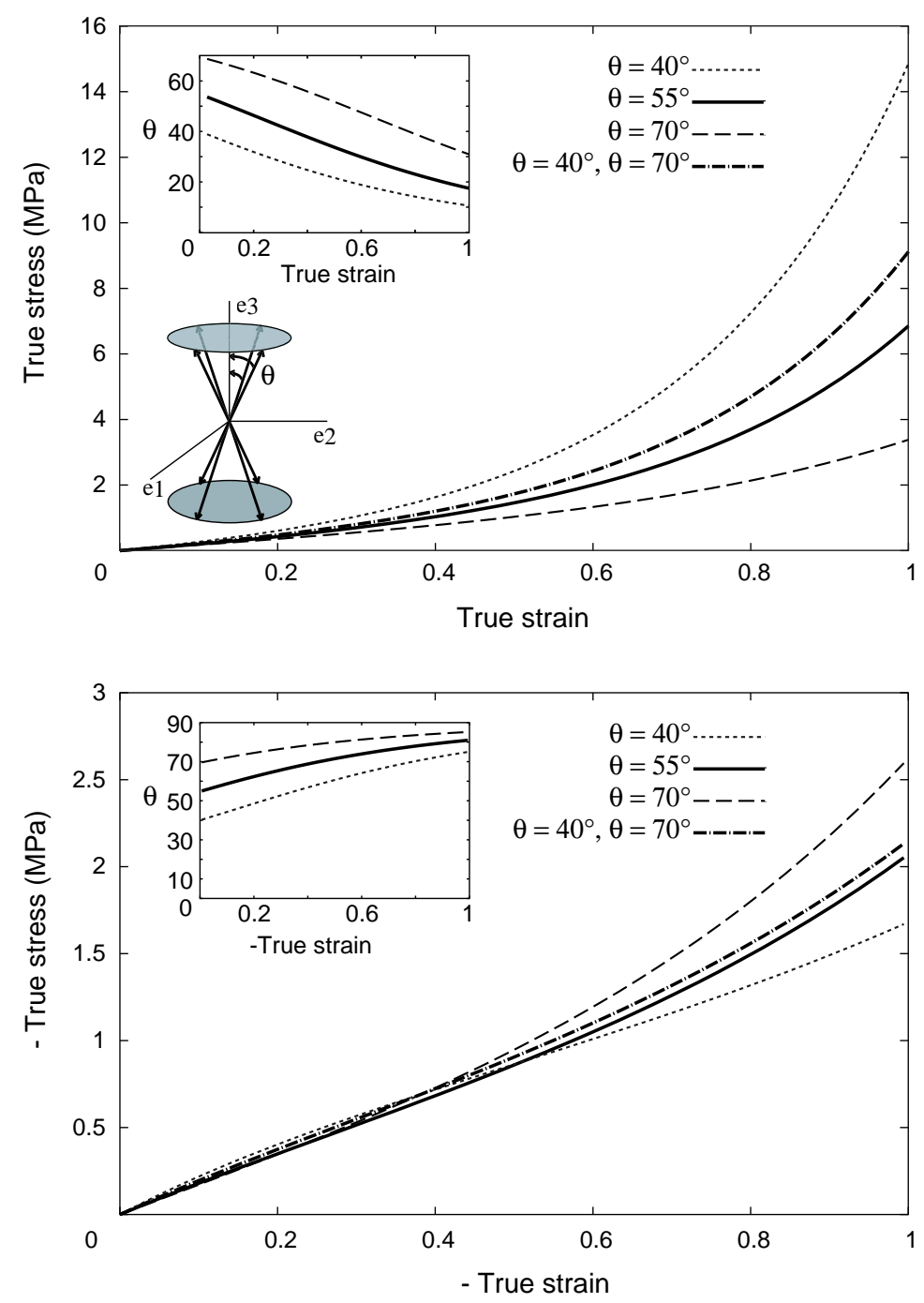

Fig. B.1. Constitutive model results for composite containing initial preferential orientation of MWNTs for uniaxial tension (a) and uniaxial compression (b). The initial orientation of MWNTs are given by the table B.1 and represented by a "cone" (a). 


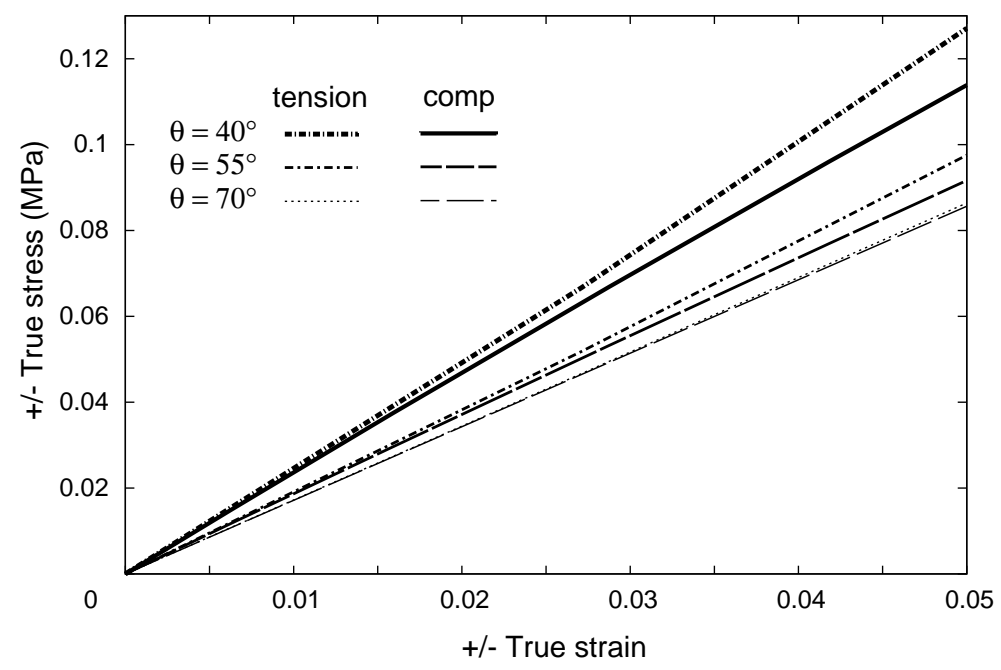

Fig. B.2. Small strain behavior as a function of the initial alignment of the fibers for uniaxial tension and uniaxial compression. 